\section{Avaliação da gestão municipal do Programa Nacional de Alimentação Escolar nos maiores municípios de Santa Catarina, Brasil}

\author{
Evaluation of municipal management of the \\ Brazilian National School Nutrition Program in \\ the largest cities of Santa Catarina State, Brazil
Evaluación de la gestión municipal del Programa de Alimentación Escolar Brasileño en las principales ciudades de Santa Catarina, Brasil

Cristine Garcia Gabriel 1

Maria Cristina Marino Calvo 1

Francisco de Assis Guedes de Vasconcelos 1 Josimari Telino de Lacerda 1

Sérgio Fernando Torres de Freitas 1

Bethsáida de Abreu Soares Schmitz ${ }^{1}$
${ }^{1}$ Centro de Ciências da Saúde, Universidade Federal de Santa Catarina, Florianópolis, Brasil.

Correspondência C. G. Gabriel

Departamento de Nutrição, Centro de Ciências da Saúde, Universidade Federal de Santa Catarina.

Campus Universitário Reitor João David Ferreira Lima, Florianópolis, SC 88040-900, Brasil. criggabriel@yahoo.com.br

\begin{abstract}
This article reports on an evaluative study of municipal management of the Brazilian National School Nutrition Program in the largest cities in Santa Catarina State, Brazil. The matrix of indicators included two dimensions: political-organizational, with the sub-dimensions resources, inter-sector action, and social control, and operational-technical, organized in nutritional and feeding efficacy, nutritional monitoring, and educational activities for a healthy diet. A total of 22 indicators were determined, and data were collected through interviews with nutritionists. The political-organizational dimension showed $60 \%$ of fair cases and $40 \%$ of poor cases, while the operational-technical dimension showed $90 \%$ of poor cases, resulting from low performance in monitoring and educational activities. Although the cases showed unsatisfactory results, the evaluation provides important data on the management of the Brazilian $\mathrm{Na}$ tional School Nutrition Program. The study emphasizes the importance of evaluation for alerting managers to the relevance of reviewing and upgrading activities.
\end{abstract}

School Feeding; Food Security; Nutrition Program and Policies

\section{Resumo}

Pesquisa avaliativa sobre a gestão municipal do Programa Nacional de Alimentação Escolar (PNAE) nos dez maiores municípios de Santa Catarina, Brasil. A matriz de indicadores contempla duas dimensões: político-organizacional (DPO), com as subdimensões recursos, atuação intersetorial e controle social; e técnico-operacional (DTO), com as subdimensões eficácia alimentar e nutricional, monitoramento alimentar e nutricional, e atuação pedagógica para a alimentação saudável. Foram elencados 22 indicadores e os dados foram coletados por meio de entrevistas com nutricionistas do PNAE. A DPO resultou em $60 \%$ dos casos regulares e $40 \%$ ruins, e a DTO obteve $90 \%$ dos casos ruins, consequência do baixo desempenho no monitoramento $e$ na atuação pedagógica. Embora os casos avaliados tenham resultados pouco satisfatórios, a avaliação oferece dados importantes sobre a gestão do PNAE. Ressalta-se a importância de avaliações com esse caráter como possibilidade de alertar os gestores para a revisão e qualificação das ações.

Alimentação Escolar; Segurança Alimentare Nutricional; Programas e Políticas de Nutrição e Alimentação 


\section{Introdução}

Secretarias Municipais de Educação (SME) são responsáveis pela gestão e execução do Programa Nacional de Alimentação Escolar (PNAE) em nível local. As normativas do programa incorporam princípios e diretrizes focados na segurança alimentar e nutricional, demandando ações articuladas e integradas $1,2,3$.

A carência de avaliações é considerada fator limitante para a qualificação do papel dos gestores ${ }^{3,4}$. Este trabalho avalia a gestão municipal do PNAE nos maiores municípios de Santa Catarina, Brasil, independentemente da modalidade de execução do programa e com foco nas ações direcionadas aos escolares do ensino fundamental.

\section{Métodos}

Pesquisa avaliativa que objetivou aferir o cumprimento da função institucional da gestão municipal para efetivação do PNAE. A construção, fundamentação e aplicabilidade do modelo avaliativo já foram descritas, demonstrando viabilidade na utilização dos indicadores 4 .

A Tabela 1 apresenta o modelo de classificação para avaliação da gestão. Os 22 indicadores e os itens observados (medidas) 4 foram considerados com o mesmo grau de importância e classificados em "bom”, "regular" ou "ruim”.

Regras para avaliação dos indicadores: (i) com uma medida - a classificação é a mesma da medida; (ii) com duas medidas - "bom" quando atinge $50 \%$ de bom nas medidas sem nenhum ruim, "ruim" quando atinge $50 \%$ de ruim sem nenhum bom, "regular" nas demais situações; (iii) com três ou mais medidas - "bom" quando atinge $50 \%$ de bom sem nenhum ruim, "ruim" quando atinge mais que $50 \%$ de ruim no somatório das medidas, "regular" nas demais situações. Nos casos em que a medida era no formato de checklist, os itens eram binários ("sim" ou "não"). Cada checklist foi avaliado como "bom" quando o somatório de "sim" para os itens resultava em valor $\geq 75 \%$, e "ruim" quando $<75 \%$.

Foram adotados escores de classificação para os indicadores (0 quando classificados como "ruim", 5 para "regular" e 7 para "bom”). As subdimensões, dimensões e a gestão foram classificadas utilizando-se as mesmas regras (Tabela 1).

O modelo foi aplicado nos dez maiores municípios catarinenses. Realizou-se coleta de dados documentais relativos ao ano de 2011 e entrevista com os nutricionistas responsáveis técnicos (RT) pelo PNAE 4 .

Este artigo integra a pesquisa do Edital MCTSECIS/CNPq no 019/2010, aprovada pelo Comitê de Ética da Universidade Federal de Santa Catarina (no 1143/2010).

\section{Resultados}

Os municípios foram analisados em cada um dos indicadores (Tabela 2) e nas subdimensões, dimensões e gestão municipal (Tabela 3).

O número máximo de resultados positivos (bom) em um mesmo município foi 7 (32\%), fato observado em dois municípios. Quatro municípios apresentaram somente 1 indicador (4,5\%) bom e o município oito (M8) obteve o maior número de indicadores ruins (19 dos 22 indicadores).

O indicador com o melhor desempenho foi o 14 (apoio ao controle social), seguido dos indicadores 1 e 8 (participação da entidade executora na execução financeira e local para as refeições respectivamente). Os indicadores de pior classificação foram o 10 (relação escolas/unidades básicas de saúde - UBS) e o 19 (vigilância).

Nos Recursos, apenas cinco municípios disponibilizaram os dados financeiros. Nesses, o porcentual de investimento em alimentos da entidade executora em relação ao recurso federal superou o desejável (> 50\%) (variação de 69\% a $379 \%$ ). O saldo anual em quatro casos variou de $1,6 \%$ a $15,2 \%$ (dois com saldos inferiores a $10 \%$, considerado positivo). Apenas três municípios apresentaram o quadro de nutricionistas adequado 5 e somente sete forneceram dados de manipuladores de alimentos (média de 158 refeições/manipulador/dia, com variação de 109 a 230). A qualificação profissional ficou regular em $90 \%$ dos casos que referiram formações periódicas para os manipuladores, mas sem cursos introdutórios de formação. As condições para o trabalhador ficaram ruins em $60 \%$ dos casos. Quanto aos nutricionistas, apenas quatro casos referiram salário base acima do piso nacional de referência, seis disponibilizavam plano de saúde e três plano de carreira. Somente um caso fornecia plano de carreira e dois plano de saúde para manipuladores. As condições para o trabalho ficaram ruins em 90\% dos casos, com insuficiência geral de equipamentos de avaliação nutricional, materiais educativos e softwares computacionais. Para os manipuladores, sete locais tinham periodicidade para exames médicos e a minoria referiu uniformes $(n=4)$ e equipamentos de proteção individual apropriados $(n=1)$. As condições para armazenamento e preparo dos alimentos não estavam boas em nenhum município, com poucos apresentando manual de boas práticas, procedimentos operacionais padronizados e fichas técnicas de preparação. O local para as 
Tabela 1

Modelo de classificação para avaliação da gestão municipal do Programa Nacional de Alimentação Escolar (PNAE).

\begin{tabular}{|c|c|c|c|c|c|}
\hline \multirow[t]{2}{*}{ Subdimensão } & \multirow[t]{2}{*}{ Indicador } & \multicolumn{4}{|c|}{ Juízo } \\
\hline & & Indicador & Subdimensão & Dimensão & Gestão \\
\hline \multicolumn{6}{|l|}{$\begin{array}{l}\text { Dimensão } \\
\text { político- } \\
\text { organizacional }\end{array}$} \\
\hline Recursos & $\begin{array}{l}\text { 1. Participação da entidade executora na } \\
\text { execução financeira do PNAE }\end{array}$ & $\begin{array}{l}\text { Bom (7) } \\
\text { Regular (5) } \\
\text { Ruim (0) }\end{array}$ & & & \\
\hline & $\begin{array}{l}\text { 2. Adequação do quadro de } \\
\text { nutricionistas }\end{array}$ & $\begin{array}{l}\text { Bom (7) } \\
\text { Ruim (0) }\end{array}$ & & & \\
\hline & $\begin{array}{l}\text { 3. Adequação da equipe de produção } \\
\text { das refeições }\end{array}$ & $\begin{array}{c}\text { Bom (7) } \\
\text { Regular (5) } \\
\text { Ruim (0) }\end{array}$ & & & \\
\hline & 4. Qualificação do profissional & $\begin{array}{l}\text { Bom (7) } \\
\text { Regular (5) } \\
\text { Ruim (0) }\end{array}$ & 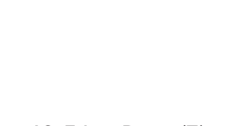 & & \\
\hline & 5. Condições para o trabalhador & $\begin{array}{l}\text { Bom (7) } \\
\text { Regular (5) } \\
\text { Ruim (0) }\end{array}$ & $\begin{array}{c}48-56=\text { Bom (7) } \\
22-47=\text { Regular (5) } \\
0-21=\text { Ruim (0) }\end{array}$ & & \\
\hline & 6. Condição para o trabalho & $\begin{array}{l}\text { Bom (7) } \\
\text { Regular (5) } \\
\text { Ruim (0) }\end{array}$ & & & \\
\hline & $\begin{array}{l}\text { 7. Condições adequadas para o } \\
\text { armazenamento e preparo dos } \\
\text { alimentos } \\
\text { 8. Local adequado para as refeições }\end{array}$ & $\begin{array}{l}\text { Bom (7) } \\
\text { Regular (5) } \\
\text { Ruim (0) } \\
\text { Bom (7) } \\
\text { Regular (5) } \\
\text { Ruim (0) }\end{array}$ & & $\begin{array}{c}19-21=\text { Bom (7) } \\
8-18=\text { Regular (5) } \\
0-7=\text { Ruim (0) }\end{array}$ & $\begin{array}{c}12-14=\text { Bom (7) } \\
\text { 6-11 = Regular (5) } \\
\text { 0-5 = Ruim (0) }\end{array}$ \\
\hline $\begin{array}{l}\text { Atuação } \\
\text { intersetorial }\end{array}$ & $\begin{array}{l}\text { 9. Articulação do PNAE com o setor } \\
\text { saúde }\end{array}$ & $\begin{array}{l}\text { Bom (7) } \\
\text { Ruim (0) }\end{array}$ & & & \\
\hline & $\begin{array}{l}\text { 10. Relação escolas - unidades básicas } \\
\text { de saúde } \\
\text { 11. Estímulo ao desenvolvimento } \\
\text { regional para a produção de alimentos }\end{array}$ & $\begin{array}{l}\text { Bom (7) } \\
\text { Ruim (0) } \\
\text { Bom (7) } \\
\text { Regular (5) } \\
\text { Ruim (0) }\end{array}$ & $\begin{array}{c}19-21=\text { Bom (7) } \\
8-18=\text { Regular }(5) \\
0-7=\text { Ruim }(0)\end{array}$ & & \\
\hline $\begin{array}{l}\text { Controle } \\
\text { social }\end{array}$ & $\begin{array}{l}\text { 13. Perfil da atuação do Conselho da } \\
\text { Alimentação Escolar } \\
\text { 14. Apoio institucional ao controle social }\end{array}$ & $\begin{array}{c}\text { Bom (7) } \\
\text { Regular (5) } \\
\text { Ruim (0) } \\
\text { Bom (7) } \\
\text { Regular (5) } \\
\text { Ruim (0) } \\
\text { Bom (7) } \\
\text { Ruim (0) }\end{array}$ & $\begin{array}{l}19-21=\text { Bom (7) } \\
\begin{array}{l}\text { 8-18 }=\text { Regular (5) } \\
0-7=\text { Ruim (0) }\end{array}\end{array}$ & & \\
\hline
\end{tabular}

(continua) 
Tabela 1 (continuação)

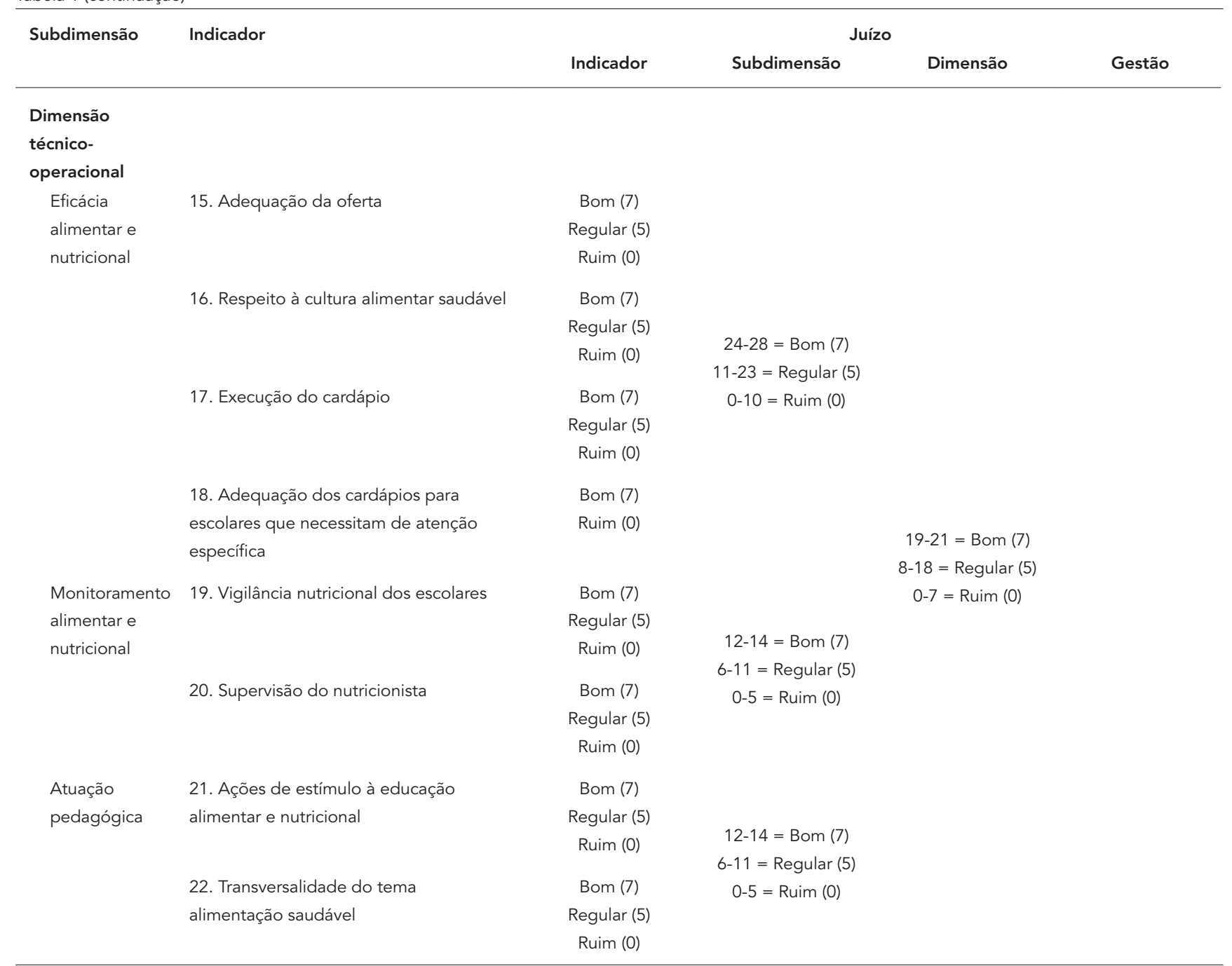

refeições era bom em $40 \%$ dos municípios que referiram refeitórios adequados na rede.

Na Atuação Intersetorial somente um município relatou articulação do PNAE com a saúde, e nenhum possuía rotina de encaminhamento de escolares para UBS. Dos cinco municípios que haviam concluído a prestação de contas, quatro empregaram $\geq 30 \%$ dos recursos federais com a agricultura familiar 1,2, entretanto, somente $60 \%$ referiram ações para apoiar o pequeno produtor.

Em Controle Social, somente dois Conselhos de Alimentação Escolar (CAEs) realizaram $\geq 10$ reuniões/ano com quórum (média de três reuniões/ano). Três locais não realizaram visitas em escolas/ano e nos demais observou-se uma média de sete visitas/ano. Somente três CAEs deliberaram sobre aspectos do Programa.

Na Eficácia Alimentar e Nutricional, a oferta ficou predominantemente ruim. Observou-se ausência de orientações sobre porcionamento. Apenas três casos incluíam frutas/hortaliças diariamente nos cardápios; quatro ofereciam salsicha/linguiça, margarina, achocolatado e pudim/ creme doce mais de uma vez/semana ou quatro vezes/mês; seis possuíam informações nutricionais dos cardápios e, embora sete municípios tenham referido cantinas comerciais, nenhum direcionava esforços para qualificá-las. O respeito à cultura alimentar foi referido por $80 \%$ dos casos, porém nenhum tinha informações sobre os hábitos alimentares dos estudantes. A execução do cardápio ficou regular em $80 \%$ dos casos. Apesar de nenhum contar com mecanismos de controle acerca das substituições de cardápios escolares, oito negaram prejuízo nutricional (controle indireto pelo estoque dos gêneros). Quanto às refeições servidas, em seis casos os manipuladores faziam a contagem. Entretanto, o dado nem 


\section{Tabela 2}

Resultados da avaliação da gestão municipal do Programa Nacional de Alimentação Escolar (PNAE) segundo indicadores, por municípios. Santa Catarina, Brasil, 2011.

\begin{tabular}{|c|c|c|c|c|c|c|c|c|c|c|c|c|c|}
\hline \multirow[t]{2}{*}{ Subdimensão } & \multirow[t]{2}{*}{ Indicador } & \multirow[t]{2}{*}{ M1 } & \multirow[t]{2}{*}{ M2 } & \multirow[t]{2}{*}{ M3 } & \multirow[t]{2}{*}{ M4 } & \multirow[t]{2}{*}{ M5 } & \multirow[t]{2}{*}{ M6 } & \multirow[t]{2}{*}{ M7 } & \multirow[t]{2}{*}{ M8 } & \multirow[t]{2}{*}{ M9 } & \multirow[t]{2}{*}{ M10 } & \multicolumn{2}{|c|}{$\begin{array}{l}\text { Municípios que } \\
\text { atingiram "bom" }\end{array}$} \\
\hline & & & & & & & & & & & & $\mathrm{n}$ & $\%$ \\
\hline \multicolumn{14}{|l|}{ Dimensão político-organizacional } \\
\hline \multirow{8}{*}{ Recursos } & 1 & & & & & & & & & & & 4 & 40 \\
\hline & 2 & & & & & & & & & & & 3 & 30 \\
\hline & 3 & & & & & & & & & & & 2 & 20 \\
\hline & 4 & & & & & & & & & & & 0 & 0 \\
\hline & 5 & & & & & & & & & & & 1 & 10 \\
\hline & 6 & & & & & & & & & & & 0 & 0 \\
\hline & 7 & & & & & & & & & & & 0 & 0 \\
\hline & 8 & & & & & & & & & & & 4 & 40 \\
\hline \multirow[t]{3}{*}{ Atuação intersetorial } & 9 & & & & & & & & & & & 1 & 10 \\
\hline & 10 & & & & & & & & & & & 0 & 0 \\
\hline & 11 & & & & & & & & & & & 3 & 30 \\
\hline \multirow[t]{3}{*}{ Controle social } & 12 & & & & & & & & & & & 2 & 20 \\
\hline & 13 & & & & & & & & & & & 1 & 10 \\
\hline & 14 & & & & & & & & & & & 8 & 80 \\
\hline \multicolumn{14}{|l|}{ Dimensão técnico-operacional } \\
\hline \multirow[t]{4}{*}{ Eficácia alimentar e nutricional } & 15 & & & & & & & & & & & 0 & 0 \\
\hline & 16 & & & & & & & & & & & 0 & 0 \\
\hline & 17 & & & & & & & & & & & 0 & 0 \\
\hline & 18 & & & & & & & & & & & 3 & 30 \\
\hline Monitoramento alimentar & 19 & & & & & & & & & & & 0 & 0 \\
\hline e nutricional & 20 & & & & & & & & & & & 0 & 0 \\
\hline \multirow[t]{2}{*}{ Atuação pedagógica } & 21 & & & & & & & & & & & 0 & 0 \\
\hline & 22 & & & & & & & & & & & 1 & 10 \\
\hline \multirow{2}{*}{ Total de "bom" por município } & $\mathrm{n}$ & 7 & 7 & 5 & 4 & 4 & 2 & 1 & 1 & 1 & 1 & & \\
\hline & $\%$ & 32 & 32 & 23 & 18 & 18 & 9 & 4,5 & 4,5 & 4,5 & 4,5 & & \\
\hline
\end{tabular}

Legenda:

Bom

- Regular

- Ruim

sempre era fidedigno e/ou sistematizado. Em quatro municípios planejava-se as quantidades de gêneros conforme o censo escolar, não tendo o número de refeições efetivamente consumidas, com adaptações conforme o estoque ou solicitações escolares. A oferta para escolares que necessitam de atenção específica foi considerada boa em três casos que possuíam cardápios para os principais grupos de estudantes com necessidades especiais. Os demais disponibilizavam somente alguns itens (leite de soja ou biscoitos sem glúten).

Em Monitoramento Alimentar e Nutricional, somente um caso possuía dados de avaliação nu- tricional dos estudantes, coletados pelo Programa Saúde na Escola (PSE) e por universidades. Entretanto, tais dados ainda não eram utilizados no PNAE. A realização de visitas mensais de nutricionistas em cada escola só se concretizou nos cinco municípios com maior número de profissionais. Nos demais as visitas ocorriam sem planejamento, visando solucionar problemas.

A Atuação Pedagógica também teve um baixo desempenho. Nove casos relataram inexistência de projetos educativos que envolvessem extensivamente os escolares e, todos referiram ausência de controle e/ou contatos sistemáticos com os atores escolares, não planejando ações 
Tabela 3

Resultados da avaliação da gestão municipal do Programa Nacional de Alimentação Escolar (PNAE) segundo subdimensão, dimensão e gestão municipal. Santa Catarina, Brasil, 2011.

\begin{tabular}{|c|c|c|c|c|c|c|c|c|c|c|c|}
\hline Dimensão & Subdimensão & M1 & M2 & M3 & M4 & M5 & M6 & M7 & M8 & M9 & M10 \\
\hline \multirow[t]{4}{*}{ Dimensão Político-Organizacional (DPO) } & Recursos & & & & & & & & & & \\
\hline & Atuação intersetorial & & & & & & & & & & \\
\hline & Controle social & & & & & & & & & & \\
\hline & DPO & & & & & & & & & & \\
\hline \multirow[t]{5}{*}{ Dimensão Técnico-Operacional (DTO) } & Eficácia alimentar e nutricional & & & & & & & & & & \\
\hline & Monitoramento alimentar e & & & & & & & & & & \\
\hline & nutricional & & & & & & & & & & \\
\hline & Atuação pedagógica & & & & & & & & & & \\
\hline & DTO & & & & & & & & & & \\
\hline \multicolumn{12}{|l|}{ Gestão } \\
\hline \multicolumn{12}{|l|}{ Legenda: } \\
\hline \multicolumn{12}{|l|}{ Bom } \\
\hline Regular & & & & & & & & & & & \\
\hline Ruim & & & & & & & & & & & \\
\hline
\end{tabular}

conjuntas. Somente dois casos possuíam o tema alimentação no currículo e apenas dois capacitaram professores em educação alimentar e nutricional.

Ao se analisar o desempenho dos municípios nas subdimensões, o Controle Social obteve o maior número de resultados bons. Recursos não houve nenhum bom, com sete casos regulares e três ruins. Eficácia Alimentar e Nutricional ficou regular em metade dos municípios e ruim no restante. As demais subdimensões apresentaram os piores desempenhos (Tabela 3).

Esses dados refletem na avaliação final. A Dimensão Político-Organizacional (DPO) resultou em $60 \%$ dos casos regulares e $40 \%$ ruins e a Dimensão Técnico-Operacional (DTO) obteve resultados piores $-90 \%$ dos casos ruins, devido ao desempenho muito baixo em Monitoramento e Atuação Pedagógica. Assim, a avaliação final da gestão apontou $90 \%$ dos municípios como ruins e um regular (Tabela 3).

\section{Discussão}

Os resultados são pouco animadores. Na DPO, aspectos relevantes devem ser aprimorados nos Recursos, destacando-se o quantitativo de profissionais e as condições de trabalho. Observaram-se discrepâncias entre as condições ofertadas em diferentes vínculos empregatícios em um mesmo município, só sendo consideradas como situações boas aquelas que contemplavam todos os trabalhadores. Ainda, evidenciaram-se algumas tendências por parte das gestões conforme a modalidade de execução do PNAE, demandando aprofundamento. Na Atuação Intersetorial, observou-se ausência de articulação dos gestores do PNAE com a saúde, considerada ação estratégica. Por outro lado, a aquisição de alimentos da agricultura familiar aparece como um ponto positivo. No Controle Social verificou-se avanços, porém um perfil pouco deliberativo dos CAEs.

Na DTO, a Eficácia Alimentar e Nutricional destaca a necessidade da gestão cumprir as normativas que orientam os cardápios e implementar ferramentas de controle que forneçam dados sobre as refeições. As duas últimas subdimensões foram as mais carentes em termos de ações. Esses dados refletem na avaliação final da gestão, a qual apontou $90 \%$ dos municípios como ruins.

Corroborando, Mello et al. 6 apontam que atividades que incluem o monitoramento nutricional dos beneficiários e ações educativas são ainda pouco efetivadas no PNAE, refletindo inadequações no planejamento e gestão do programa.

Ressalta-se a necessidade ainda presente de avaliações normativas e estudos que identifiquem as causas e consequências desse desempenho. Mesmo com os achados pouco satisfatórios, ressalta-se a importância da manutenção desses indicadores como possibilidade de alertar e estimular os gestores na revisão das ações. Ainda, destaca-se a escassez de estudos com essa perspectiva, colocando o modelo avaliativo como possibilidade de contribuir para a gestão do PNAE. 


\section{Resumen}

Investigación evaluativa sobre la gestión municipal del Programa de Alimentación Escolar Brasileño (PNAE) en las diez mayores ciudades de Santa Catarina, Brasil. La matriz contempla dos dimensiones: político-organizacional (DPO), con las subdimensiones recursos, acción intersectorial y control social y técnico-operacional (DTO), con la eficacia alimentaria y nutricional, monitoreo alimentaria y nutricional y acción pedagógica para una alimentación saludable. Fueron listados 22 indicadores y os dados fueron colectados a través de entrevistas con los nutricionistas. El DPO se ha traducido en $60 \%$ de los casos regulares y el $40 \%$ malos, mientras DTO consiguió $90 \%$ de los casos malos, consecuencia del bajo desempeño en el monitoreo y en la acción pedagógica. Aunque los casos evaluados hayan resultados poco satisfactorios, la evaluación muestra datos importantes sobre la gestión. Se resalta la importancia de las evaluaciones como la posibilidad de alertar a los gestores para la calificación de las acciones.

Alimentación Escolar; Seguridad Alimentaria;

Programas y Políticas de Nutrición y Alimentación

\section{Referências}

1. Brasil. Lei no 11.947, de 16 de junho de 2009. Dispõe sobre o atendimento da alimentação escolar e do Programa Dinheiro Direto na Escola aos alunos da educação básica. Diário Oficial da União 2009; 17 jun.

2. Fundo Nacional de Desenvolvimento da Educação. Resolução no 38, de 16 de julho de 2009. http:// www.fnde.gov.br/programas/alimentacao-esco lar/alimentacao-escolar-legislacao (acessado em 13/Jun/2014)

3. Belik W, Chaim NA. O Programa Nacional de Alimentação Escolar e a gestão municipal: eficiência administrativa, controle social e desenvolvimento local. Rev Nutr 2009; 22:595-607.

4. Gabriel CG, Calvo MCM, Ostermann RM, Vasconcelos FAG. Proposta e aplicabilidade de modelo para avaliação da gestão municipal do Programa Nacional de Alimentação Escolar. Cad Saúde Pública 2014; 30:1731-44.

\section{Colaboradores}

C. G. Gabriel e M. C. M. Calvo contribuíram com a concepção do projeto e análise e interpretação dos dados, redação do artigo e aprovação final da versão a ser publicada. F. A. G. Vasconcelos, J. T. Lacerda, S. F. T. Freitas e B. A. S. Schmitz contribuíram com a concepção do projeto e análise e interpretação dos dados, revisão crítica do artigo e aprovação final da versão a ser publicada.

\section{Agradecimentos}

A todos os gestores envolvidos e, em especial, aos nutricionistas do PNAE pela disponibilidade e interesse com a pesquisa. Ao CNPq; (processo no 559328/2010-9) pelo financiamento da investigação.
5. Conselho Federal de Nutricionistas. Resolução CFN no 465, de 23 de agosto de 2010. http://www. cfn.org.br/novosite/arquivos/Resol-CFN-465-atri buicao-nutricionista-PAE.pdf (acessado em 12/ Jun/2014).

6. Mello AL, Vidal Júnior PO, Sampaio LR, Santos LAS, Freitas MCS, Fontes GAV. Perfil do nutricionista do Programa Nacional de Alimentação Escolar na Região Nordeste do Brasil. Rev Nutr 2012; 25:119-32.

Recebido em 09/Jun/2013

Versão final reapresentada em 15/Jun/2014 Aprovado em 03/Jul/2014 